\title{
A scintillation counter consisting of a pure CsI crystal, WLS and APD for Belle II
}

\section{H. Aihara}

The University of Tokyo, 7-3-1 Hongo Bunkyo-ku, Tokyo 113-0033, Japan

\section{A. Epifanov}

Budker Institute of Nuclear Physics, akademika Lavrentieva prospect, Novosibirsk, 630090,

Russian Federation

\section{Y. Jin*}

The University of Tokyo, 7-3-1 Hongo Bunkyo-ku, Tokyo 113-0033, Japan

E-mail: jin@hep.phys.s.u-tokyo.ac.jp

\section{K. Wan}

The University of Tokyo, 7-3-1 Hongo Bunkyo-ku, Tokyo 113-0033, Japan

Belle II, as the upgrade of Belle, aims to search for New Physics with 40 times higher luminosity. In order to cope with such a high luminosity, an electromagnetic calorimeter using pure CsI scintillation crystals, whose scintillation time are around 30 nanoseconds, has been proposed. Silicon avalanche photodiodes (APD), making up for the low light yield of pure CsI, are considered as photosensors. The application of the innovative wavelength shifter (WLS) that perfectly matches the emission spectrum of pure CsI and the quantum efficiency of APD maximizes the light collection. The equivalent noise energy of the counter is determined using cosmic rays.

38th International Conference on High Energy Physics 3-10 August 2016

Chicago, USA

\footnotetext{
* Speaker.
} 


\section{Introduction}

In the last decade, fruitful achievements have emerged at Belle, a dedicated particle physics experiment operated at KEKB accelerator, the world's highest luminosity machine at that time, investigating CP-violation effects in B meson decays at the High Energy Accelerator Research Organization (KEK) in Tsukuba, Japan. Not only has Belle revealed the mystical inner of the $\mathrm{CP}$-violation, but also excited the curiosity of scientists and driven them to chase the tail of New Physics. Cater to people's urge for a deeper understanding of the nature, Belle collaboration decided to upgrade Belle into Belle II, targeting at a 40 times higher luminosity. Via such an increase on luminosity and the subsequent unprecedentedly huge amount of data, some hints to New physics could be obtained [1].

At the electromagnetic calorimeter (ECL) of Belle detector, the combination of PIN - photodiodes and thallium-doped CsI crystals had accomplished a marvellous task. However, in order to cope with a more challenging condition at Belle II, it is necessary to conceive new approaches, especially for the endcap of ECL. Taken into account the large backgrounds which would render the notable pile-up noise of Belle II, pure CsI scintillation crystals whose scintillation time are around 30 nanoseconds have been proposed for the upgrade of the endcap of ECL [2]. Silicon avalanche photodiode (APD), a compact device that possesses remarkable insensitivity to magnetic fields, requires relatively low bias voltages and has a low dark current at the operating point, has been considered as a candidate of photosensor. Moreover, APD's ability of rapidly amplifying a weak light signal into a large electrical signal makes up for the low light yield of pure CsI, which entitles the scheme under consideration to very competitive advantages.

As a calorimeter, precise measurement of particle energy is the key point. Previously, some thorough studies [3] [4] have proven that the mentioned scheme, along with a wavelength shifter (WLS), can reach the required noise level of Belle II ( $0.5 \mathrm{MeV}$ per counter). Afterwards, extensive research was carried out to further reduce the electronic noise of this scintillation counter. In this paper, the result of employing a new shaper plus FADC module is presented.

\section{Experimental setup}

A pure CsI crystal in truncated pyramidal shape, $30 \mathrm{~cm}$ in length, with a $5 \mathrm{~cm} \times 5 \mathrm{~cm}$ front surface and a $6 \mathrm{~cm} \times 6 \mathrm{~cm}$ rear surface, produced by Kharkov Company, Ukraine, is used in this counter as scintillator. A wavelength shifter $(6 \mathrm{~cm} \times 6 \mathrm{~cm} \times 0.5 \mathrm{~cm})$ [5] from LumInnoTech LLC is attached to the rear surface of the crystal. Both are wrapped by a $200 \mu \mathrm{m}$ layer of Gore-Tex teflon and encapsulated in a $40 \mu \mathrm{m}$ aluminised mylar envelope. Four Hamamatsu S8664-55 APDs are coupled to the central area of the WLS via the optical grease OKEN 6262A. The light collection is enhanced [3] thanks to the application of WLS that perfectly matches the emission spectrum of pure CsI (peak at $315 \mathrm{~nm}$ ) and the quantum efficiency of APD (over 0.8 in the range from $500 \mathrm{~nm}$ to 850 $\mathrm{nm}$, however, only 0.3 at $315 \mathrm{~nm}$ ) [6]. The electronic readout system is comprised of Hamamatsu S8664-55 APDs, a CAEN A1422 preamplifier and a readout module designed by Budker Institute of Nuclear Physics (BINP), as shown in Fig. 1. The BINP module is a CR-4RC shaper of $30 \mathrm{~ns}$ shaping time plus a 12-bit flash analog to digital converter (FADC) of $40 \mathrm{MHz}$ sampling frequency. Bias voltages are provided by Hamamatsu C 8238 voltage supply. A series of periodic rectangular 


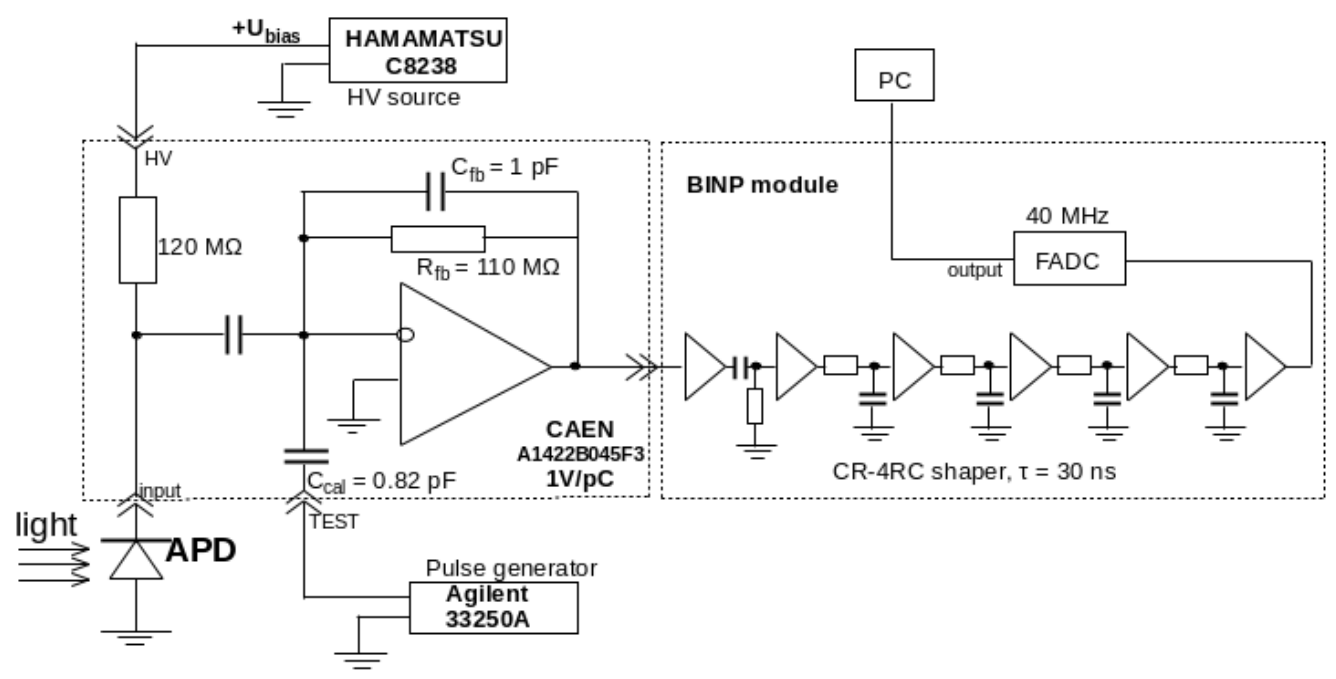

Figure 1: Schematic diagram of the electronic readout chain.

voltage signals is fed to the test channel of CAEN amplifier by the pulse generator Agilent 33250A. The root mean square of the output signal from FADC is a measure of the electronic noise, in units of ADC counts. The whole measurement is conducted in a temperature box at $25.0 \pm 0.1{ }^{\circ} \mathrm{C}$.

\section{Measurement of equivalent noise energy}

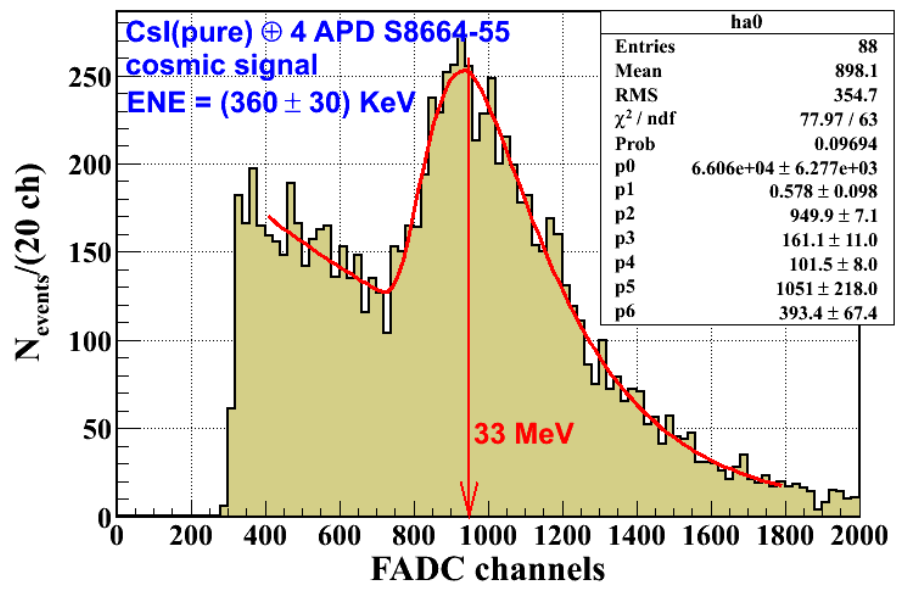

Figure 2: Amplitude spectrum of the signal from cosmic muons in the counter. Fit function is shown by red curve.

In order to evaluate the electronic noise in units of energy, a calibration by cosmic muons was performed. Monte Carlo simulation was implemented to derive the most probable energy deposition of cosmic muons passing through the given scintillator crystal. It is assumed that scintillation photons are emitted uniformly and isotropically among the passage of the muon traversing the 
crystal. Optical refractions and reflections are parameterized according to the optical properties of the scintillator and wrapping materials. The angular flux of cosmic muons is set by applying the approximate extrapolation formula 29.4 in PDG [7]:

$$
\frac{d I_{\mu}}{d E_{\mu} d \Omega} \approx \frac{0.14 E_{\mu}^{-2.7}}{c m^{2} \operatorname{sr~GeV}} \times\left\{\frac{1}{1+\frac{1.1 E_{\mu} \cos \theta}{115 \mathrm{GeV}}}+\frac{0.054}{1+\frac{1.1 E_{\mu} \cos \theta}{850 \mathrm{GeV}}}\right\} .
$$

And the deposited energy is integrated by the Bethe Equation (Eq 33.5 in PDG). The most probable energy was found to be $33 \mathrm{MeV}$. The observed cosmic ray spectrum was fitted by the sum of an exponential function and a Logarithmic Gaussian function, as shown in Fig. 2. By identifying the peak of the spectrum as $33 \mathrm{MeV}$ and assuming the linearity, the ADC count was converted into energy. As a result, the equivalent noise energy of the counter is found to be $360 \pm 30 \mathrm{keV}$.

\section{Conclusion}

The upgrade of accelerator KEKB into SuperKEKB has been almost completed. The first currents were already traced through the new collider. Meanwhile, upgrade of Belle II is still ongoing and scheduled to be finished in 2018. Hamamatsu APD S8664-55, together with the WLS specially developed by LumInnoTech LLC, has provided a promising option to serve as the readout system for the endcap of Belle II ECL. The counter studied here, a nearly finalized scheme with 4 pieces of S8664-55 APD, has reached a sufficiently low noise level.

\section{References}

[1] T. Aushev, W. Bartel, A. Bondar, et al., Physics at Super B Factory, arXiv:1002.5012, 2010.

[2] T. Abe, et al., Belle II technical design report, arXiv:1011.0352, 2010.

[3] Y. Jin, H. Aihara, O. V. Borshchev, D. A. Epifanov, S. A. Ponomarenko, N. M. Surin, Study of a pure CsI crystal readout by APD for Belle II endcap ECL upgrade, Nucl. Instrum. Meth. A 824 (2016): 691-692.

[4] H. Aihara, O. Borshchev, D. Epifanov, Y. Jin, S. A. Ponomarenko and N. M. Surin, Study of scintillation counter consisting of a pure CsI crystal and APD, PoS(PhotoDet2015) 052.

[5] http://www.luminnotech.com/

[6] http://www.hamamatsu.com/jp/en/S8664-55.html

[7] http://pdg.lbl.gov 